\title{
The picosecond structure of ultra-fast rogue waves
}

\section{Avi Klein, Shir Shahal, Gilad Masri, Hamootal Duadi, Kfir Sulimani, et al.}

Avi Klein, Shir Shahal, Gilad Masri, Hamootal Duadi, Kfir Sulimani, Ohad Lib, Hadar Steinberg, Stanislav A. Kolpakov, Moti Fridman, "The picosecond structure of ultra-fast rogue waves," Proc. SPIE 10517, Real-time Measurements, Rogue Phenomena, and Single-Shot Applications III, 1051704 (15 February 2018); doi: 10.1117/12.2285987

SPIE. Event: SPIE LASE, 2018, San Francisco, California, United States 


\title{
The picosecond structure of ultra-fast rogue waves
}

\author{
Avi Klein ${ }^{1}$, Shir Shahal ${ }^{1}$, Gilad Masri ${ }^{1}$, Hamootal Duadi ${ }^{1}$, Kfir Sulimani ${ }^{2}$, Ohad Lib ${ }^{2}$, Hadar \\ Steinberg ${ }^{2}$, Stanislav A. Kolpakov ${ }^{3}$, and Moti Fridman ${ }^{1}$ \\ ${ }^{1}$ Faculty of Engineering and the Institute of Nanotechnology and Advanced Materials, Bar Ilan \\ University, Ramat Gan 5290002, Israel \\ ${ }^{2}$ The Racah Institute of Physics, the Hebrew University of Jerusalem, Israel \\ ${ }^{3}$ School of Engineering and Applied Science, Aston University, Aston Triangle, Birmingham B4 \\ 7ET, UK
}

\begin{abstract}
We investigated ultrafast rogue waves in fiber lasers and found three different patterns of rogue waves: singlepeaks, twin-peaks, and triple-peaks. The statistics of the different patterns as a function of the pump power of the laser reveals that the probability for all rogue waves pattens increase close to the laser threshold. We developed a numerical model which prove that the ultrafast rogue waves patterns result from both the polarization mode dispersion in the fiber and the non-instantaneous nature of the saturable absorber. This discovery reveals that there are three different types of rogue waves in fiber lasers: slow, fast, and ultrafast, which relate to three different time-scales and are governed by three different sets of equations: the laser rate equations, the nonlinear Schrodinger equation, and the saturable absorber equations, accordingly. This discovery is highly important for analyzing rogue waves and other extreme events in fiber lasers and can lead to realizing types of rogue waves which were not possible so far such as triangular rogue waves.
\end{abstract}

Keywords: Extreme events, rogue waves, optical data processing, four-wave mixing

\section{INTRODUCTION}

Rogue waves which are also called freak waves are an example for extreme events with higher probability than expected by stochastic models. ${ }^{1}$ Such waves appear in numerous fields, including hydrodynamics, atomic physics, optics, and lasers.

We investigated ultrafast rogue waves which are created in fiber lasers. We focused on their temporal structure and measured the rogue wave structure as a function of the laser power and state of polarization.

We also developed numerical models and extended them to include the non instantaneous response of the saturable absorber and the two components of the vector field. These models reveal that the underlying mechanism of ultrafast rogue waves is different then what was consider so far.

\section{TIME LENS}

In order to investigate the temporal structure of ultrafast rogue waves, we resorted to a time-lens with 500 fs resolution. ${ }^{2-5}$ A time-lens is based on the temporal equivalent of an imaging lens in space. In spatial optics, a light propagates distance $u$ from an object to a lens, and then an image is generated at a distance $v$ from the lens. The equivalent temporal scheme of such a lens is a time-lens, where a signal is propagating in dispersive material a distance $u$ until it reaches a highly nonlinear fiber where we impose on it a quadratic phase shift. Then, after propagating a distance $v$ in more dispersive material, we obtain an image of the input signal. The image can be a magnified copy or a shrinkage copy according to the parameters of the time-lens and according to the dispersion values before and after the time-lens.

Further author information: (Send correspondence to M.F.)

M.F.: E-mail: mordechai.fridman@biu.ac.il, Telephone: 97235317524

Real-time Measurements, Rogue Phenomena, and Single-Shot Applications III, edited by

Bahram Jalali, Daniel R. Solli, Günter Steinmeyer, Proc. of SPIE Vol. 10517, 1051704

(C) 2018 SPIE · CCC code: $0277-786 X / 18 / \$ 18 \cdot$ doi: 10.1117/12.2285987

Proc. of SPIE Vol. 10517 1051704-1 


\section{GAIN MODULATIONS: SLOW ROGUE WAVES}

Any fluctuation in a laser power is being compensated by a pump depletion leading to a negative feed-back, until the equilibrium is restored. This is due to the coupling between the power of the output light, and the value of the gain in the cavity. This can be understood by resorting to the laser rate equations as: ${ }^{6}$

$$
\frac{\partial E(t)}{\partial t}=\frac{1}{\tau_{c}}(G(t)-\alpha) E(t)+i \omega E(t)
$$

were $E(t)$ is the electric field in the cavity, $\tau_{c}$ is the cavity life-time, $G(t)$ is the gain as a function of time, $\alpha$ is the losses, and $\omega$ is the light frequency. The gain as a function of time follows:

$$
\frac{\partial G(t)}{\partial t}=\frac{1}{\tau_{f}}\left(P-G(t)-|E(t)|^{2} G(t)\right)
$$

were $\tau_{f}$ is the fluorescence life-time, and $P$ is the pump power of the laser. Evaluating these equations leads to a relaxation oscillations of the cavity due to any change in the pump power, as:

$$
\omega_{r o}=\frac{1}{\sqrt{\tau_{c}^{2}+\tau_{f}^{2}}} .
$$

When modulating the pump power at frequencies which are close or larger than $\omega_{\text {ro }}$ a chaotic behavior can arise. From this chaotic intensity fluctuations, rare peaks with high intensity appears which are considered as rogue waves. However, these rogue waves have the time-scale of the relaxation oscillations of the laser which is at the order of milliseconds to microseconds.

\section{SOLITONS COLLISIONS: FAST ROGUE WAVES}

Solitons are a special light pulses in which the nonlinearity is compensating for the dispersion in the fiber. In such cases a soliton pulse can propagate in the cavity with out dispersion and preserve its shape. The equation describing the propagation of pulses in fiber which combines the nonlinear interaction and the dispersion of the light in the fiber is the nonlinear Schroedinger equation (NLSE):

$$
i \frac{\partial u}{\partial z}+\frac{1}{2} \frac{\partial^{2} u}{\partial t^{2}}+|u|^{2} u=0
$$

were $u$ is the field envelope of the pulse traveling in the fiber. The solution for this equation is: ${ }^{7}$

$$
u(z, t)=\Sigma_{k=1}^{N} u_{k}(z, t),
$$

were $u_{k}(z, t)$ is the single soliton solution, namely:

$$
u_{k}(z, t)=2 \nu_{k} \operatorname{sech}\left(2 \nu_{k}\left(t-\xi_{k}\right)\right) e^{i 2 \mu_{k}\left(t-\xi_{k}\right)+i \delta_{k}},
$$

were $\mu$ is the soliton amplitude, $\xi$ is the soliton temporal delay, $\mu$ is the speed of the soliton, and $\delta$ is the phase of the soliton. When solving these equations with two solitons, we obtain that two soliton will interact with each other. A force between the two solitons will either pull them together or push them apart according to the relative phase between them. In addition, the interaction between the solitons can lead to power transfer from one soliton to another and to collisions between solitons, in which the solitons changes the speed due to the collision.

This complicated dynamics of many solitons interacting together, leads to rare events in which many soliton collide and generate a high peak. These high peaks are considered as rogue waves and their time-scales is the time-scale of the solitons. 


\section{ULTRA-FAST ROGUE WAVES}

We tried modeling a fiber laser, but could not get the ultrafast rogue waves which were measured in the experiment. Therefore, we extended current models to include the polarization mode dispersion in the cavity and the non-instantaneous response of the saturable absorber. Only when including both phenomena, we numerically observed the same rogue waves as measured in the experiment.

Specifically, to simulate the laser, we extended the numerical model presented in. ${ }^{8}$ The propagation of the vector field envelope was simulated in each element of the fiber laser and repeated over large number of round trips. The field envelope

$$
\vec{\psi}=\left\{\psi_{x}, \psi_{y}\right\}
$$

traveling in a dispersive nonlinear fiber is governed by the non-linear Schrdinger equation:

$$
i \vec{\psi}_{z}+\frac{D}{2} \vec{\psi}_{t t}+|\vec{\psi}|^{2} \vec{\psi}=0
$$

where $D$ is the dispersion tensor of the fiber with different values for each component of the field due to PMD, $z$ is the propagation distance, and $t$ is time in a moving frame of reference with the group velocity.

The EDF is simulated by:

$$
i \vec{\psi}_{z}+\frac{D_{2}}{2} \vec{\psi}_{t t}+\Gamma_{2}|\vec{\psi}|^{2} \vec{\psi}=\frac{i g_{0}}{1+Q / Q_{s a t}}\left(\vec{\psi}+\beta_{2} \vec{\psi}_{t t}\right)
$$

where $Q_{\text {sat }}$ represents the saturation energy, $g_{0}$ is the small signal gain, $\beta_{2}$ the spectral width of the gain, $\Gamma_{2}$ is the nonlinear coefficient, and the total energy $Q$ is:

$$
Q=\int_{-\infty}^{\infty}|\vec{\psi}|^{2} d t
$$

The instantaneous saturable absorber is modeled by:

$$
T(t)=T_{0}+\Delta T \frac{I(t)^{2}}{I_{\text {sat }}+I(t)},
$$

where $I(t)=|\vec{\psi}|^{2}, T_{0}$ is the transmission for low intensity optical field, and $I_{\text {sat }}$ is the saturation intensity. In order to include the response time of the saturable absorber, we imposed a low pass filter with a cut-off frequencies of $1 \mathrm{THz}$ on $T(t)$.

The propagation in the fiber laser follows the principle states of polarization (PSP) in the cavity while the polarization controller couples between the two PSP according to:

$$
\vec{\psi}_{\text {out }}=\left[\begin{array}{cc}
(1-\kappa) e^{i \varphi_{1}} & \kappa e^{i \varphi_{2}} \\
\kappa e^{-i \varphi_{2}} & (1-\kappa) e^{-i \varphi_{1}}
\end{array}\right] \vec{\psi}_{\text {in }}
$$

where $\kappa$ is the amplitude coupling strength, $\varphi_{1}$ is the relative phase between the two states of polarization, and $\varphi_{2}$ is the cross phase.

The full results, as well as more details on the experiments and the numerical model are described in. ${ }^{9}$

\section{CONCLUSIONS AND FUTURE PROSPECTS}

We measured the statistics of ultrafast rogue waves in fiber lasers and found that the underlying mechanism is different than what was previously considered. We will continue to investigate extreme events in different schemes, such as phase locked fiber lasers ${ }^{6,10-13}$ fiber lasers with unique states of polarization, ${ }^{14,15}$ and different types of fiber components ${ }^{16-19}$ We hope to utilize our temporal imaging to focus light through dispersive material and to create rogue waves for high resolution imaging with nano-particles. ${ }^{20-25}$ 


\section{REFERENCES}

[1] Akhmediev, N., Kibler, B., Baronio, F., Belić, M., Zhong, W.-P., Zhang, Y., Chang, W., Soto-Crespo, J. M., Vouzas, P., Grelu, P., et al., "Roadmap on optical rogue waves and extreme events," Journal of Optics 18(6), 063001 (2016).

[2] Klein, A., Yaron, T., Preter, E., Duadi, H., and Fridman, M., "Temporal depth imaging," Optica 4(5), 502-506 (2017).

[3] Klein, A., Shahal, S., Masri, G., Duadi, H., and Fridman, M., "Four wave mixing-based time lens for orthogonal polarized input signals," IEEE Photonics Journal 9(2), 1-7 (2017).

[4] Yaron, T., Klein, A., Duadi, H., and Fridman, M., "Temporal superresolution based on a localization microscopy algorithm," Applied Optics 56(9), D24-D28 (2017).

[5] Arnon, S. and Fridman, M., "Data center switch based on temporal cloaking," Journal of Lightwave Technology 30(21), 3427-3433 (2012).

[6] Fridman, M., Nixon, M., Ronen, E., Friesem, A. A., and Davidson, N., "Phase locking of two coupled lasers with many longitudinal modes," Optics letters 35(4), 526-528 (2010).

[7] Armaroli, A., Conti, C., and Biancalana, F., "Rogue solitons in optical fibers: a dynamical process in a complex energy landscape?," Optica 2, 497-504 (May 2015).

[8] Soto-Crespo, J., Grelu, P., and Akhmediev, N., "Dissipative rogue waves: extreme pulses generated by passively mode-locked lasers," Physical Review E 84(1), 016604 (2011).

[9] Klein, A., Masri, G., Duadi, H., Sulimani, K., Lib, O., Steinberg, H., Kolpakov, S. A., and Fridman, M., "Ultrafast rogue waves patterns in fiber lasers," submitted to Phys. Rev. Lett. .

[10] Nixon, M., Fridman, M., Ronen, E., Friesem, A. A., and Davidson, N., "Phase locking of two fiber lasers with time-delayed coupling," Optics letters 34(12), 1864-1866 (2009).

[11] Eckhouse, V., Fridman, M., Davidson, N., and Friesem, A. A., "Phase locking and coherent combining of high-order-mode fiber lasers," Optics letters 33(18), 2134-2136 (2008).

[12] Ronen, E., Fridman, M., Nixon, M., Friesem, A., and Davidson, N., "Phase locking of lasers with intracavity polarization elements," Optics letters 33(20), 2305-2307 (2008).

[13] Fridman, M., Eckhouse, V., Davidson, N., and Friesem, A. A., "Simultaneous coherent and spectral addition of fiber lasers," Optics letters 33(7), 648-650 (2008).

[14] Fridman, M., Nixon, M., Dubinskii, M., Friesem, A. A., and Davidson, N., "Fiber amplification of radially and azimuthally polarized laser light," Optics letters 35(9), 1332-1334 (2010).

[15] Fridman, M., Nixon, M., Grinvald, E., Davidson, N., and Friesem, A. A., "Real-time measurement of space-variant polarizations," Optics express 18(10), 10805-10812 (2010).

[16] Shahal, S., Klein, A., Masri, G., and Fridman, M., "Fused fiber micro-knots," Applied optics 55(17), 45384541 (2016).

[17] Shahal, S., Klein, A., Masri, G., Duadi, H., and Fridman, M., "Long period fiber gratings with off-resonance spectral response based on mechanical oscillations," JOSA A 34(2), 264-269 (2017).

[18] Masri, G., Shahal, S., Klein, A., Duadi, H., and Fridman, M., "Polarization dependence of asymmetric off-resonance long period fiber gratings," Optics express 24(26), 29843-29851 (2016).

[19] Shahal, S., Duadi, H., and Fridman, M., "High-order modes micro-knot excited by a long-period fiber grating," Sensors 17(11), 2490 (2017).

[20] Abu-Ghosh, S., Fixler, D., Dubinsky, Z., and Iluz, D., "Continuous background light significantly increases flashing-light enhancement of photosynthesis and growth of microalgae," Bioresource technology 187, 144148 (2015).

[21] Ankri, R., Ashkenazy, A., Milstein, Y., Brami, Y., Olshinka, A., Goldenberg-Cohen, N., Popovtzer, A., Fixler, D., and Hirshberg, A., "Gold nanorods based air scanning electron microscopy and diffusion reflection imaging for mapping tumor margins in squamous cell carcinoma," ACS nano 10(2), 2349-2356 (2016).

[22] Abu-Ghosh, S., Fixler, D., Dubinsky, Z., and Iluz, D., "Energy-input analysis of the life-cycle of microalgal cultivation systems and best scenario for oil-rich biomass production," Applied Energy 154, 1082-1088 (2015). 
[23] Meir, R., Betzer, O., Motiei, M., Kronfeld, N., Brodie, C., and Popovtzer, R., "Design principles for noninvasive, longitudinal and quantitative cell tracking with nanoparticle-based ct imaging," Nanomedicine: Nanotechnology, Biology and Medicine 13(2), 421-429 (2017).

[24] Polak, P., Zalevsky, Z., and Shefi, O., "Gold nanoparticles-based biosensing of single nucleotide dna mutations," International journal of biological macromolecules 59, 134-137 (2013).

[25] Ankri, R. and Fixler, D., "Gold nanorods based diffusion reflection measurements: current status and perspectives for clinical applications," Nanophotonics 6(5), 1031-1042 (2017). 